\title{
Perspectives on UK Care Quality Commission's Inspections of Primary Care Practices -
}

\author{
Results of an online survey conducted by the BAPIO GP Forum
}

\begin{abstract}
Background- In the middle of a pandemic, the health and social care services were experiencing their worst challenges, and staff faced burnout. Professionals working in primary care raised concerns about the UK Care Quality Commission's inspection regime, especially its effectiveness, impact on services, equality, diversity, and inclusion.
\end{abstract}

Methods -An online anonymised survey was carried out in July 2021 and received 130 responses, the majority from the GP partners but also locum and sessional colleagues as well as other members of the general practice team.

Results - About $80 \%$ of respondents reported having a good-outstanding rating. However, only $20 \%$ felt that their experience of the CQC inspections was positive. More than $85 \%$ of respondents felt that the inspections did not add value to clinical care or prevent harm, were intense and took staff away from direct clinical care.

$80 \%$ felt that the inspections were not fair, transparent, objective, or replicable and found them traumatic rather than constructive. More than $50 \%$ felt that practices led by Black, Asian and minority ethnic (BAME) General Practitioners, small practices in inner-city areas, and rural practices in areas of deprivation received disproportionately poorer outcomes.

Almost $86 \%$ of respondents felt that the inspectors demonstrated insights into the systemic challenges faced by primary care. About half of the respondents did not feel supported by the Local Medical Committees and their Clinical Care Group.

Discussion \& Conclusion - The findings demonstrated that the inspections were not considered practical or constructive and took the general practice team away from patient care. Of particular concern was that perception that the system was unfair and discriminatory for BAME GPs small practices and those in the inner city or rural locations with multiple deprivations. The authors urge the CQC to ensure that the profession is supported and that any inspection process is fair, non-discriminatory and improves patient care.

\section{Keywords}

The Care Quality Commission, General Practice, Primary Care, Inspections, Patient safety, Patient harm, BAME, Bias
Kamal Sidhu', Kalindi

Tumurogoti ${ }^{1}$, Naureen

Bhatti $^{2}$, Abrar Hussein $^{3}$ \&

Indranil Chakravorty ${ }^{4}$

1 GP, British Association of Physicians of Indian Origin, General Practice Forum

2 GP, Strategic Lead for Workforce Race Equality and Differential Attainment in Primary Care, HEE London

3 Consultant Psychiatrist, Berkshire Healthcare NHS Foundation Trust, UK 4 Consultant Physician, Professor, Faculty of Life \& Medical Sciences, University of Hertfordshire \& Chair, Bapio Institute for Health Research, Bedford, UK

Correspondence to sidhuks@gmail.com

Cite as: Sidhu, K.S., Tumurogoti, K., Bhatti, N., Hussein, A., Chakravorty, I. (2022) Perspectives on UK Care Quality Commission's inspections of primary care practices. The Physician Vol 7; Issue 2: 1-9 ePub 30.01.22 DOI https://doi.org/10.38192/1.7.2.7

Article Information Submitted 22.01.22 Revised 28.01.22 Published 30.01.22

ISSN 2732-513X (Print) ISSN 2732 - 5148 (Online) ठacts 


\section{Background}

The Care Quality Commission (CQC) was set up as an independent regulator of all health and social care providers in the United Kingdom, taking over this role from its predecessor, the Health Care Commission. Its values include excellence, caring (treating everyone with dignity and respect), integrity and teamwork. Its ambitions include protecting the rights of vulnerable people, listening to experiences, and working with organisations and public groups.[1] The CQC is committed to tackling organisational and health inequalities across all aspects of its regulatory process, so the CQC must have protocols and processes that are transparent and fair.

In March 2021, the Royal College of General Practitioners Council, listening to the complaints from the majority of its members, voted to ask the CQC to undertake an independent review of all practices that had been de-registered or found to be inadequate in the last five years. The RCGP Council raised concerns about the perceived unfair treatment of practices with majority Black Asian and minority ethnic staff (BAME), single-handed practices, or those located in socio-economic deprivation. [2] The CQC were quick to reject the demand for an independent inquiry but did agree to an internal review on the impact of its processes on BAME GP led practices.

The British Association of Physicians of Indian Origin (BAPIO), a national, voluntary organisation representing many doctors and nurses from the Indian subcontinent, has been championing the cause for equality, diversity and inclusion in healthcare. BAPIO argues that this is essential for excellence in patient care and fostering a supportive workplace. Among its constituents, the BAPIO General Practice Forum (GPF) focussed on the support of primary care professionals. In the summer of 2021, BAPIO GPF designed and conducted an online survey to ascertain the experiences and perspectives of primary care professionals through its national network on inspections by the CQC.

\begin{abstract}
Aims
To explore the experiences of primary care professionals on fairness, the impact of inspections and support offered by the local commissioning care groups (CCGs)
\end{abstract}

\section{Methods}

An anonymous online survey was designed and conducted using Survey Monkey software (B) distributed through the BAPIO membership lists social network groups and cascaded to connected groups. The GPF conducted the Survey over two weeks in August 2021. The questionnaire and responses received are available online to review here. The results were analysed using simple proportions and distributions.

\section{Results}

\section{Respondents}

There were 130 responses received, including 92 General Practitioners and 38 primary care professionals, including Nurses and practice managers. There were 41 who completed the ethnicity question.

\section{Inspections}

$79 \%$ of respondents had been inspected by the CQC in the previous four years, $20 \%$ more than four years ago, and one had not been inspected.

\section{Outcomes}

Of the respondents, $5 \%$ were judged outstanding, $77 \%$ 'good', see figure 1 . There were 19 practices 'requiring improvement', three inadequate and two declined to disclose the rating received. 
Q3

\section{What was your most recent CQC rating?}

Answered: 130 Skipped: 0

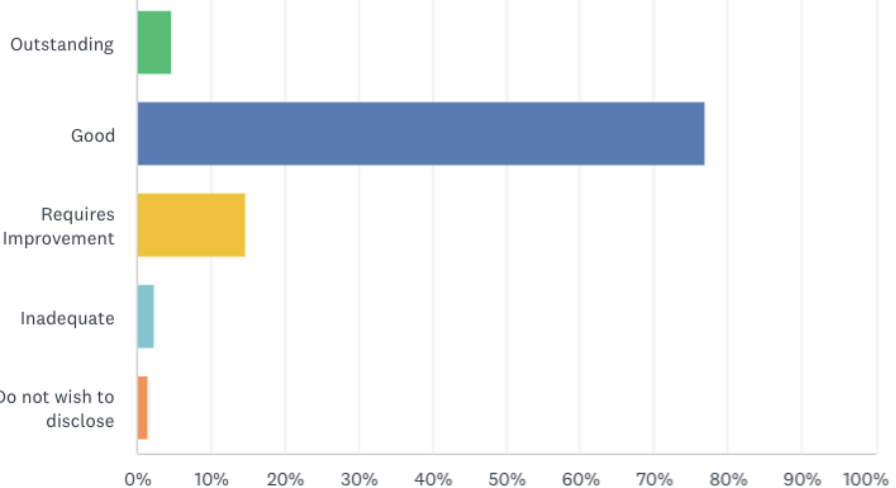

The overall experience of Inspections

There were $25(19 \%)$ respondents who felt their experience was positive, and $68(48 \%)$ felt it was negative or strongly negative figure 2 .

Q4

What was your overall experience of the CQC inspection?

Answered: 129 Skipped: 1

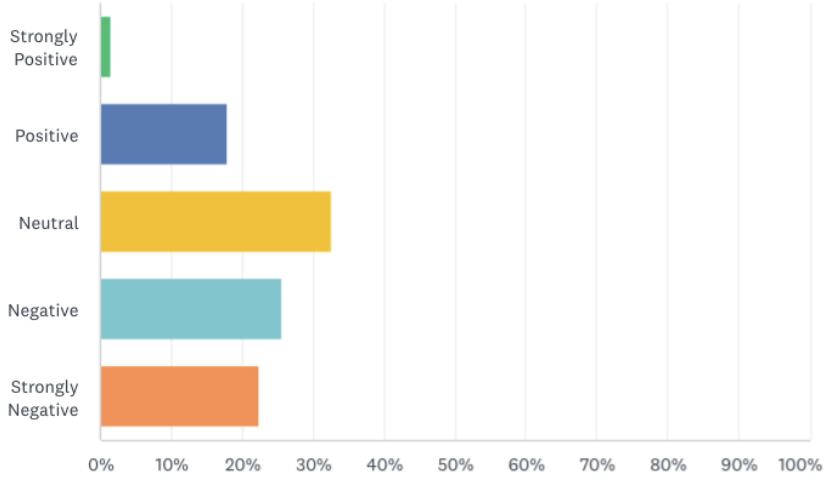

Impact of Inspections on patient care

Seventy-three per cent of respondents felt the workload in undergoing the inspection was heavy and took them away from providing patient care. At the same time, $21 \%$ agreed the workload was heavy but did not affect patient care.

Experience with the Inspection team

Only over a quarter of respondents agreed that their experience with the inspection team was pleasant, while $38 \%$ described their experience as unpleasant or very unpleasant.

Factors affecting the rating received

A majority of respondents felt that their ethnicity, being a single/ multiple handed practice, working in areas of social deprivation and their inner-city/ rural location impacted the rating received, figure 3 . 
Do you think the following factors have a bearing on your Inspections \& rating?

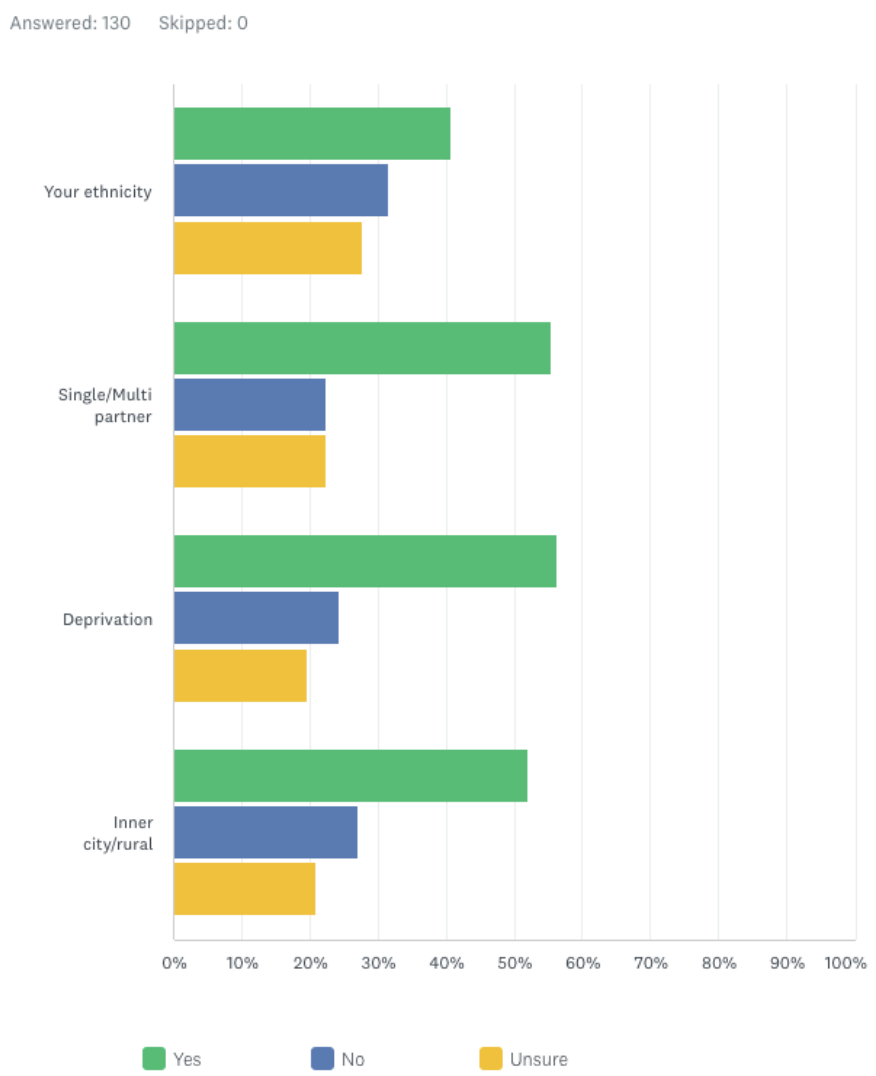

Fairness \& systemic context

Eighty per cent of respondents did not feel that the inspection was fair, transparent, objective and replicable. Sixty-six per cent of respondents did not feel that the inspection took cognisance of the systemic challenges, figure 4.

CQC inspectors demonstrated insights into the systemic challenges faced by you as a Practice

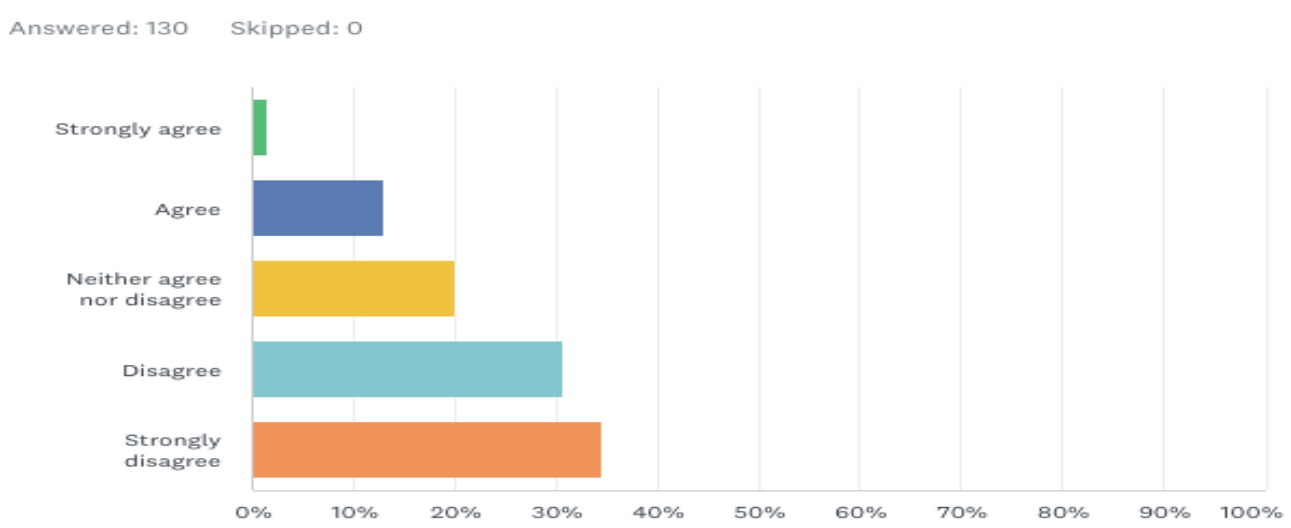


Insight into diversity and cultural context

Sixty per cent of respondents disagreed that the inspection team demonstrated insight into diversity or cultural aspects of their patients or staff, figure 5 .

\section{The CQC inspectors demonstrated insight into the diversity and cultural aspects of the team and patient population}

Answered: 130 Skipped: 0

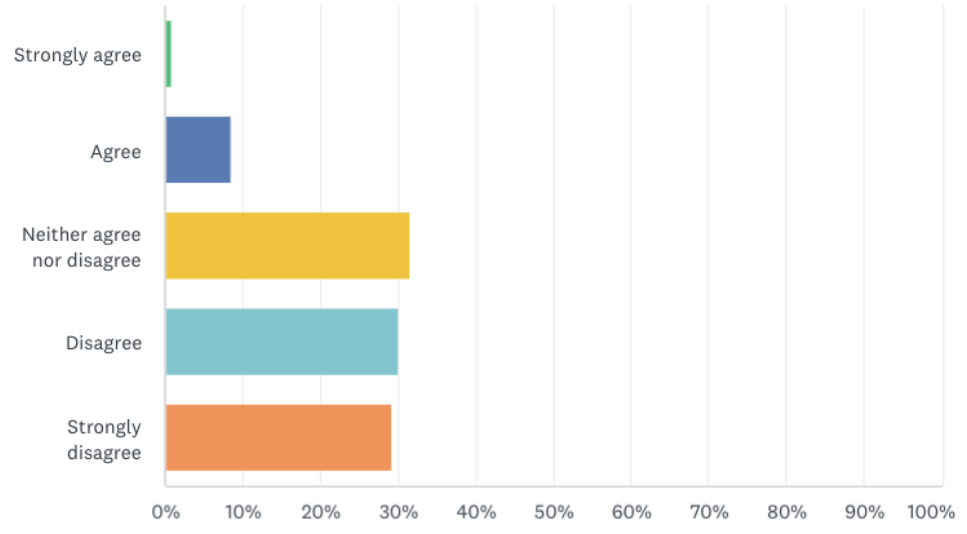

In receiving support from local negotiating committees and the Commissioning Care Groups, 30\% reported in the affirmative, but $60 \%$ disagreed that they had received support.

Feedback and responsiveness

Thirty-five per cent of respondents felt the need to challenge the inspection findings but did not feel they could or that their views would be considered.

Reflect patient care and prevent harm

Whether the inspections were a true reflection of the care provided and whether they contributed to patient safety or prevention of harm, $71-80 \%$ of respondents disagreed.

$52 \%$ of respondents agreed that practices with BAME GPs received disproportionately poorer outcomes, 48\% neither agreed nor disagreed, and only one respondent disagreed. Over $75 \%$ agreed that the experience of the inspection and the team was traumatic rather than constructive.

\section{Discussion}

The CQC was created to ensure that healthcare providers were fit for purpose and its stated mission is to protect the public, including the vulnerable. The CQC aspires to be excellent, caring, and demonstrate integrity and teamwork as an organisation. An analysis of 6313 inspection reports of NHS GP Practices published on the CQC website reveals; 312 (5\%) rated as outstanding, $5723(91 \%)$ as good, 148 $(2.3 \%)$ requiring improvement and $33(0.5 \%)$ as inadequate. If the inspections are fit for purpose, this data demonstrates that most primary care practices inspected by the CQC provide good to outstanding care. Our dataset is comparable to the overall picture of most respondents from practices rated as good to outstanding $(79 \%)$, and only a tiny minority were inadequate $(2.3 \%)$.

\section{Reduction in harm/ Patient Safety}

Despite most respondents reporting their rating as good to outstanding, the data reveals that a substantial proportion felt the workload required during the inspection was intense and took them away from patient care. They also felt that the inspection was not constructive, did not reflect the care provided and did not contribute to patient safety or reduce harm. Protecting patients, particularly the vulnerable, is the primary function of the inspections, making this perception significant and putting the imperative back on the CQC to demonstrate that inspections lead to harm reduction. Analysis by the King's Fund showed evidence of anticipatory impact: 
the CQC set expectations of quality, and providers responded to these in advance of an inspection.

Inspections had some positive effects (e.g., it helped some organisations to prioritise quality issues) and some adverse effects (e.g., some providers focused much energy on getting through the inspection process rather than on improving the quality of care). [3] There was scant evidence of systemic impact in metrics such as maternity services and primary care prescribing. Intelligent monitoring of a range of performance indicators (IM datasets) had little or no correlation with the subsequent ratings of general practices, highlighting the limitations of risk-based regulatory models that use routinely reported performance data to target regulatory interventions.[3,4]

Few studies have demonstrated whether inspections improve the quality of care. A systematic review found no relation between the inspection process and increased quality. [5] Interrupted time-series studies suggest CQC inspections were not associated with changes in care quality measures. Although some statistically significant changes were present, the size of the effect was considered unlikely to be clinically relevant. [6] The opportunity cost of a CQC inspection is estimated to lie between $£ 169,691$ and $£ 418,136$ in 2018.

Our data showed that the experience of interactions with the inspection team was less than optimal and over a third felt this to be unpleasant. In the King's Fund research, one of the most striking findings was that the relationships between CQC staff and health and social care professionals and managers fundamentally affect the way regulation works and its impact, contributing to the variation in providers' experiences inspection.[3]

\section{Equality \& Diversity}

Our data confirmed concerns raised by RCGP on three counts; that the inspection outcomes are disproportionately worse for GPs from BAME backgrounds, those in single-handed or small partnerships and those who operated in socioeconomically deprived areas. In 2021, the CQC proclaimed its values of ensuring culturally appropriate care for its registrants, sensitive to people's cultural identity or heritage - being alert and responsive to beliefs or conventions determined by cultural heritage.[7] Our data demonstrated that the GP practices did not believe that the CQC Inspectors considered the ethnocultural diversity of patients and staff during the inspections or the outcomes.

Roger Kline undertook a qualitative review of CQC's action plan in response to Workforce Race Equality Standards data from 2016-17, demonstrating an inherent bias in the organisation. Evidence from interviews with staff confirmed substantial disenchantment with the unfairness of opportunities for career progression leading to a high turnover of BAME staff. [8] We analysed a subset of 33 practices reported as being 'inadequate' in the CQC published reports (2022- see Appendix 1). Our analysis revealed that BAME GPs predominantly managed $79 \%$, $74 \%$ were single-handed or had less than three partners, and $45 \%$ were in the lowest three deciles of the Multiple Deprivation Index ranking (2019).

Perceptions from our Survey (the majority of who were from practices with good to outstanding ratings), the WRES data, and our analysis of CQC inspection reports - showed that the majority of 'inadequate' practices shared three factors (BAME, less than three partners and a location in multiple deprivations). This analysis raises the possibility of institutional bias. It puts the onus on the CQC to provide evidence to the contrary or take decisive action to achieve equality, diversity, and inclusion in its leadership, staff, governance, and practices.

\section{Conclusion}

The COVID-19 pandemic has pushed an already stretched, under-resourced, understaffed and overburdened healthcare system to its brink. This stress manifests in all health and social care areas, but the impact is most strongly felt in the primary and social care sector. The population has widespread disenchantment and demoralised healthcare staff demonstrate discontent and burn-out. In addition, there has been a considerable rise in awareness of the inherent inequalities affecting people's lives and manifest in disproportionate levels of mortality and morbidity.

The authors welcome the $\mathrm{CQC}$ review of the impact and experience of CQC regulation on ethnic minority-led practices and hope our survey results add to the data on understanding the lived experience of ethnic minority GPs. 
References

1 Who we are | Care Quality Commission. https://www.cqc.org.uk/about-us/ourpurpose-role/who-we-are (accessed 22 Jan 2022).

2 College calls on Care Quality Commission for action to address impact of inspections on BAME GPs. https://www.rcgp.org.uk/aboutus/news/2021/march/college-calls-on-carequality-commission-for-action-to-addressimpact-of-inspections-on-bame-gps.aspx (accessed 22 Jan 2022).

3 Impact of the Care Quality Commission on provider performance. The King's Fund. 2018.https://www.kingsfund.org.uk/publicati ons/impact-cqc-provider-performance (accessed 22 Jan 2022).

4 CQC-style inspections don't raise standards or improve patient safety, say RCGP members The BMJ. https://www.bmj.com/content/363/bmj.k421 6 (accessed 22 Jan 2022).
5 Flodgren G, Pomey M-P, Taber SA, et al. Effectiveness of external inspection of compliance with standards in improving healthcare organisation behaviour, healthcare professional behaviour or patient outcomes. Cochrane Database of Systematic Reviews Published Online First: 2011. doi:10.1002/14651858.CD008992.pub2

6 Castro AC. Evaluating the effect of external inspections of the Care Quality Commission of acute NHS hospitals in England. 2018.https://etheses.whiterose.ac.uk/21399/ (accessed 22 Jan 2022).

7 Culturally appropriate care | Care Quality Commission.

https://www.cqc.org.uk/guidanceproviders/adult-social-care/culturallyappropriate-care (accessed 22 Jan 2022).

8 Kline R. Inequality in recruitment outcomes for Black and Minority Ethnic staff within the Care Quality Commission. ;:69. 
Appendix 1

- Unit 8, 24 Martineau Place, Birmingham, B2 4UH Provided by: $\underline{\text { Halcyon Medical }}$ Limited

- The Croft Surgery Barnham Road, Eastergate, Chichester, PO20 3RP

- Bridgnorth Road, Highley, Bridgnorth, WV16 6HG; Provided by: Highley Medical Centre

- The Surgery, Central Clinic, Hall Street, Dudley, DY2 7BX, Provided by: Dr Touseef $\underline{\text { Safdar }}$

- Hertford Road, Brighton, BN1 7GF; Provided by: School House Surgery

- The Village Surgery, 157 High Street, New Malden, KT3 4BH

- Victoria Medical Centre, 1 Queens Road, Barking, IG11 8GD; Provided by: Dr N Niranjan's Practice

- 1 Grove Road, Tottenham, London, N15 5HJ; Provided by: Dr Jerome Kaine Ikwueke

- 6 The Broadway, Castletown, Sunderland, SR5 3EX; Provided by: Dr Hesham Moustafa Koriem

- Failsworth District Centre, Ashton Road West, Failsworth, Manchester, M35 0AD; Provided by: Failsworth Group Practice

- The Hub, Shiners Way, South Normanton, Alfreton, DE55 2AA; Provided by: The Village Surgery

- 8a Patford Street, Calne, SN11 OEF; Provided by: Patford House Surgery Partnership

- Buxted Medical Centre, Framfield Road, Buxted, Uckfield, TN22 5FD; Provided by: Buxted Medical Centre

- Gosport War Memorial Hospital, Bury Road, Gosport, PO12 3PW; Provided by: Dr Carl Wyndham Robin William Anandan

- The Surgery, London Road, Teynham, Sittingbourne, ME9 9QL; Provided by: Dr Ravi Kumar

- The Centre Surgery, 29 Hill Street, Hinckley, LE10 1DS; Provided by: Hinckley \& Bosworth Medical Alliance Limited

- Muglet Lane, Maltby, Rotherham, S66 7NA; Provided by: Primary Care Today Limited

- Burnage Avenue, Clock Face, St. Helens, WA9 4QB; Provided by: Four Acre Health Centre

- 434 Altrincham Road, Baguley, Wythenshaw, Manchester, M23 9AB; Provided by: The Park Medical Centre

- 68 The Drive, Ilford, IG1 3HZ; Provided by: Dr Padma Gooty

- The Health Centre, 20 Cleveland Square, Middlesbrough, TS1 2NX; Provided by: Prospect Surgery

- Elms Road, Botley, Oxford, OX2 9JS; Provided by: Botley Medical Centre

- London Road, Stanway, Colchester, CO3 8NZ; Provided by: Dr Kamal Kumarapriya Abeysundara

- Moorside Medical Centre, 681 Ripponden Road, Oldham, OL1 4JU; Provided by: Dr Kiren Kaur

- 26 Stephenson Road, Walthamstow, London, E17 7JT; Provided by: The Firs

- Ryhall Road, Stamford, PE9 1YA; Provided by: Lakeside Healthcare Partnership

- Stirling Road, Plymouth, PL5 1PL; Provided by: Access Health Care Ltd 
- 1 Evington Lane, Leicester, LE5 5PQ; Provided by: Dr Abdul-Kader Vania

- 267 Ealing Road, Wembley, HA0 1EU; Provided by: Sudbury and Alperton Medical Centre

- Station Road, Pulham Market, Diss, IP21 4TX; Provided by: Dr Farrook Ahmed Mondol

- 2 Bata Avenue, East Tilbury, Tilbury, RM18 8SD; Provided by: Dr Reshma Rasheed

- Carpenters Practice, 236-252 High Street, London, E15 2JA; Provided by: $\underline{\text { AT Medics }}$ Limited

- 25 Street End Road, Chatham, ME5 0AA; Provided by: Stonecross and West Drive Surgery 